DATA

(DATE)
GENERE:

(GENDER)
ETÀ

$(A G E)$ 
SOSTANZA ATTESA

(EXPECTED SUBSTANCE)

HAI CONSUMATO LA SOSTANZA PRIMA DEL TEST?

Sí $\square \quad$ NO $\square$

(DID YOU CONSUME THE SUBSTANCE BEFORE THE TEST?)

(YES) (NO)

CHE RISULTATO TI ASPETTI DAL TEST COLORIMETRICO?

(WHAT RESULT DO YOU EXPECT FROM THE COLORIMETRIC TEST?)

$\square$ LA SOSTANZA CHE HO COMPRATO (THE SUBSTANCE I BOUGHT)

$\square$ UN ADULTERANTE/TAGLIO (AN ADULTERANT)

$\square$ UN'ALTRA SOSTANZA (ANOTHER SUBSTANCE)

DOPO AVER RICEVUTO INFORMAZIONI A PROPOSITO DEL DRUG CHECKING, ACCETTI DI CONSEGNARE UN PICCOLA FRAZIONE DELLA TUA SOSTANZA PER ESSERE ANALIZZATA ON SITE E SUCCESSIVAMENTE IN LABORATORIO? SÍ $\square \quad$ NO $\square$

(AFTER RECEIVING COMPLETE INFORMATION ABOUT DRUG CHECKING DO YOU AGREE TO DELIVER A SMALL FRACTION OF YOUR SUBSTANCE TO BE ANALYZED ON SITE AND LATER IN THE LABORATORY? YES $\square$ NO $\square)$

ORA CHE CONOSCI IL RISULTATO ASSUMERAI LA SOSTANZA? SÍ $\square$ NO $\square$

(NOW THAT YOU KNOW THE TEST RESULT ARE YOU GOING TO CONSUME THE SUBSTANCE? YES $\square$ NO $\square$ ) 


\section{TEST COLORIMETRICO}

(COLORIMETRIC TEST)

DESCRIZIONE CAMPIONE

(SAMPLE DESCRIPTION)

\begin{tabular}{|c|c|c|}
\hline \multirow{2}{*}{$\begin{array}{c}\text { Reagente } \\
\text { (Colorimetric Reagents) }\end{array}$} & \multirow[b]{2}{*}{ Positive } & \multirow[b]{2}{*}{ No reaction } \\
\hline & & \\
\hline \multicolumn{3}{|l|}{ Marquis } \\
\hline \multicolumn{3}{|l|}{ Mecke } \\
\hline \multicolumn{3}{|l|}{ Mandelin } \\
\hline \multicolumn{3}{|l|}{ Lieberman } \\
\hline Scott & & \\
\hline
\end{tabular}

RISULTATO DEL TEST COLORIMETRICO

(COLORIMETRIC TEST RESULT)

$\square$ Conclusivo Conclusive $\square$ Non conlusivo

Sostanza identificata

(identified substance) 